\title{
Anabases
}

ANABASES Traditions et réceptions de l'Antiquité

$11 \mid 2010$

Varia

\section{Le faux, source intentionnelle d'erreurs : le cas des contrefaçons de peintures antiques}

Delphine Burlot

\section{(2) OpenEdition}

1 Journals

Édition électronique

URL : http://journals.openedition.org/anabases/872

DOI : 10.4000/anabases.872

ISSN : 2256-9421

Éditeur

E.R.A.S.M.E.

Édition imprimée

Date de publication : 1 mars 2010

Pagination : 181-192

ISSN : 1774-4296

\section{Référence électronique}

Delphine Burlot, «Le faux, source intentionnelle d'erreurs : le cas des contrefaçons de peintures antiques ", Anabases [En ligne], 11 | 2010, mis en ligne le 01 mars 2013, consulté le 14 novembre 2019. URL : http://journals.openedition.org/anabases/872 ; DOI : 10.4000/anabases.872

(c) Anabases 


\section{Le faux, source intentionnelle d'erreurs: le cas des contrefaçons de peintures antiques}

Delphine Burlot

L'HISTOIRE D'UN OBJET DÉPEND DES ALÉAS DU TEMPS. Elle est constituée d'une succession d'événements comprenant sa fabrication, son utilisation, sa disparition et son « invention " (ou découverte) par l'archéologue. Ce dernier cherche alors à remonter le fil chronologique en s'appuyant sur toutes les informations que peut lui fournir l'objet. Il étudie les matériaux employés, l'état de conservation de l'objet, le contexte de sa découverte et il peut compléter son étude par des analyses en laboratoire. L'interprétation qu'il fait dépend des données qu'il a recueillies lors de son étude ainsi que de ses connaissances. La découverte d'un objet du passé offre donc à l'archéologue la possibilité d'en retrouver l'histoire.

Dans le cas du faux, l'histoire de l'objet précède bien souvent sa fabrication et la chronologie est inversée. Le faussaire donne une " ancienneté " à son œuvre afin de séduire l'archéologue ou le collectionneur. Il lui invente un passé antique car il souhaite que l'objet soit pris pour tel, et pour cela il s'inspire d'œuvres dont il imite le style, la technique de réalisation et la composition. Mais le faussaire invente également le reste de l'histoire de l'œuvre, et crée une fable relatant un enfouissement et une prétendue découverte. Pour appuyer sa supercherie, il fabrique de multiples preuves matérielles censées prouver l'antiquité de l'objet. Il utilise pour cela différents truchements, comme l'application de fausses patines, la réalisation de fausses cassures ou de fausses restaurations anciennes.

Rien n'est laissé au hasard dans la fabrication de la contrefaçon, ni les matériaux employés, ni le vieillissement feint de l'œuvre, ni l'invention de son histoire. Tous ces 
éléments concourent à la fabrication d'une œuvre nouvelle, unique, rare et attrayante: un faux ${ }^{1}$.

La fable racontée par le faussaire à son client participe de la supercherie et dans certains cas la "victime ", un archéologue ou un collectionneur, est connue du faussaire. Le faussaire est en effet souvent proche des archéologues, il travaille pour eux comme artiste ou comme restaurateur. Il est donc au fait des dernières découvertes, et ses connaissances de la matière lui permettent de l'imiter.

Le faux répond à une demande et il varie suivant les goûts et les modes. L'erreur qu'il produit de manière intentionnelle est la conséquence $\mathrm{du}$ " désir » antiquaire. Nous allons voir dans cet article quelques exemples de contrefaçons de peinture antique réalisées par d'habiles faussaires qui ont cherché à tromper les plus grands archéologues du $\mathrm{XVIII}^{\mathrm{e}}$ siècle, au moment où la découverte d'Herculanum provoquait l'apparition d'un véritable engouement pour la peinture des anciens Romains.

\section{Premières déposes de peintures antiques et premières contrefaçons}

Déjà bons connaisseurs des monnaies et de la sculpture antiques, les collectionneurs et les antiquaires ne semblent pas s'intéresser à la peinture antique avant le début du XVII ${ }^{e}$ siècle, car l'évolution technique qui permet de détacher les peintures ne survient que tardivement. À partir du moment où une technique de dépose est mise au point, les peintures ne sont plus laissées in situ mais elles sont extraites des " grottes " (où seuls les plus téméraires des amateurs d'antiquité pouvaient jusque là les admirer), pour être placées chez des collectionneurs, qui les exposent comme de petits tableaux ${ }^{2}$. Dès lors, l'intérêt pour ces œuvres atteint un public plus large, et les collectionneurs, désireux de faire valoir leur bien, les présentent aux visiteurs de leurs palais et les font copier par des artistes afin qu'elles puissent être diffusées ${ }^{3}$.

1 Il faut bien faire attention à ne pas confondre une copie, une réplique et un faux. La copie et la réplique consistent en la reproduction de tout ou partie d'une œuvre, sans intention délictueuse, contrairement au faux. L'artiste qui fait une copie reproduit le sujet et la composition de l'original sans forcément en imiter le style. Plusieurs peintres célèbres se sont adonnés à cet exercice La réplique est une copie réalisée par l'auteur de l'original. Il s'agit de la seconde version d'un même tableau.

2 La dépose des Noces Aldobrandini en 1601 est un événement majeur dans l'histoire de la réception de la peinture antique car elle fut à l'origine de l'engouement pour cet art. Le cardinal Aldobrandini, propriétaire de l'œuvre, l'avait incrustée dans le mur de l'un des pavillons qui se trouvait dans le jardin de sa villa à Magnanapoli, à Rome. Au sujet de cette peinture, voir G. FusCOnI, La fortuna delle Nozze Aldobrandini, (Studi e testi, 363), Vatican, 1994.

3 Pietro Santo Bartoli et Gian Pietro Bellori publièrent différents ouvrages sur ces peintures dont le plus célèbre est Le pitture antiche del Sepolcro dei Nasoni, Rome, 1680. 
Or parmi ces premiers fragments d'enduit antique conservés dans les collections privées romaines se trouvent des œuvres dont l'authenticité est douteuse. Le cardinal Barberini, qui était fier de présenter une grande peinture de paysage trouvée dans les fondations de son palais, ainsi qu'un fragment représentant la déesse Rome, trouvé sur l'Esquilin, possédait également plusieurs peintures antiques, qui, selon les visiteurs du XVIII ${ }^{\mathrm{e}}$ siècle, étaient fort "dans le goût de Raphaël ${ }^{4}$ ». Nous savons par des témoignages plus récents, auxquels nous devons nous référer car ces œuvres ont disparu aujourd'hui, qu'il s' agit d'œuvres modernes ${ }^{5}$. Il s'agit de contrefaçons, réalisées peut-être à la demande du cardinal Barberini lui-même, qui accroissait ainsi le prestige de sa collection.

Au début du XVIII ${ }^{\mathrm{e}}$ siècle, l'engouement pour la peinture antique se propage chez les voyageurs britanniques, qui découvrent ces œuvres dans les palais romains lors de leur Grand Tour, et en ramènent des copies en souvenir, voire en achètent des fragments.

L'un d'eux, le docteur Mead, médecin du roi, mais aussi collectionneur et numismate, attache rapidement beaucoup d'importance à cet art et dans les années 1740 il achète de nombreux fragments d'enduit peint retrouvés à Rome. Plusieurs d'entre eux faisaient partie de la collection du cardinal Massimi, mais d'autres étaient de provenance plus incertaine ${ }^{6}$. Ainsi, une peinture représentant Auguste et sa cour se trouvait dans l'atelier d'un peintre, Camillo Paderni, lorsqu'un voyageur britannique la vit et décida de l'acheter pour Mead ${ }^{7}$. Il est certain que Mead y attachait beaucoup d'importance, car il la montrait fréquemment à ses hôtes et dans ses dispositions testamentaires il ordonna que l'œuvre restât dans sa famille ${ }^{8}$. Malheureusement pour lui, Mead croyait détenir une des plus belles peintures de l'Antiquité alors qu'il avait été joué par un faussaire. La composition et le sujet représenté semblent bien étranges pour une peinture romaine: l'œuvre représenterait Auguste rendant sa couronne à Phraatès, comme l'a décrit Horace. On reconnaît Auguste ainsi que Mécène et Agrippa dont les portraits

4 J. Richardson, Traité de la peinture et de la Sculpture, Genève, 1972, III, p. 265.

5 Ces peintures représentaient une Vénus et trois Amours, une Parque et un Cupidon endormi. Elles furent relativement célèbres au début du XVIII ${ }^{\mathrm{e}}$ siècle. Les gravures de ces œuvres montrent des compositions très chargées, au style plus baroque qu'antique. Lucia Faedo pense que le Cupidon endormi était antique, voir L. FAEDO, "Vivere con gli antichi. Una pittura antica a Palazzo Barberini e la sua fruizione tra XVII e XVIII secolo", in L. Mochi Onori, S. Schütze, F. Solinas (éd.), I Barberini e la cultura europea del Seicento, Rome 2007. Voir aussi H. Whitehouse, Ancient Mosaics and Wallpaintings, wallpaintings, the paper museum of Cassiano dal Pozzo (The paper museum of Cassiano dal Pozzo, series A, antiquities and architecture, 1), Londres, 2001, p. 55.

$6 \quad$ Ibid., fig. 77-80.

7 L'œuvre fut achetée par Alexander Cunningham, qui voyageait en Italie avec son ami le peintre Allan Ramsay. A. Cunningham, "The fine arts at Rome in the years 1736 and 1737", Gentleman's magazine, 40 (1853), p. 240 et M. ForCellino, "La formazione e il metodo di Camillo Paderni", Eutopia, II 2 (1993), p. 49-64.

8 A. Michaelis, Ancient Marbles in Great Britain, Cambridge, 1882, p. 49, n. 27. 
sont souvent représentés sur des médailles et des intailles. Or, nous n'avons pas d'autres exemples de représentations de l'empereur entouré de sa cour en peinture romaine. De plus, la ressemblance des personnages avec leurs portraits sur les médailles, loin de nous confirmer la valeur historique de l'œuvre comme semblait le penser le docteur Mead, tendrait au contraire à nous faire douter de son authenticité. La provenance supposée de l'œuvre (le Palatin), l'histoire représentée et la trop grande similitude avec le texte d'Horace laissent penser qu'il s'agit d'une contrefaçon, réalisée dans le but de plaire à un collectionneur épris d'histoire romaine et de numismatique comme le docteur Mead.

\section{Peintures volées... ou contrefaites?}

La découverte d'Herculanum en 1738 éveilla l'intérêt des antiquaires de toute l'Europe. Pour la première fois un grand nombre de peintures antiques était mis au jour. De nombreux fragments étaient déposés afin d'être placés dans la collection du roi de Naples. Celui-ci exerçait un contrôle très strict sur les découvertes et il empêchait la diffusion d'informations sur les peintures. Il attisa ainsi la curiosité du public, avide de voir les œuvres, mais seuls quelques privilégiés étaient admis au musée de Portici. Très vite, la demande fut plus pressante, et plusieurs collectionneurs bravèrent l'interdiction du roi de Naples en achetant à Rome ou à Naples des fragments d'enduit peint provenant clandestinement d'Herculanum. Le comte de Caylus (1692-1765) reçut d'Italie un fragment représentant un guerrier grec qu'il publia rapidement dans le premier volume de son recueil d'antiquités, non sans fierté, car il est persuadé qu'elle provient

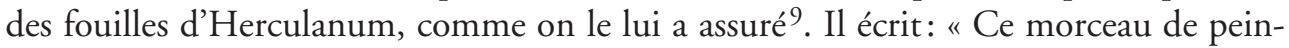
ture à fresque est recommandable par son antiquité, et par le lieu d'où il vient. Il a été trouvé dans cette ville infortunée d'Herculanum, que le Vésuve engloutit au temps de Pline. " Il remarque plus loin: «Cet ouvrage, en un mot, m’a rappelé tout ce que je connaissais des peintures Romaines ou trouvées à Rome ${ }^{10}$. "

Cela n'est pas étonnant, si l'on considère ce dessin de Bartoli copiant une peinture antique trouvée à Rome en 1684 près des Sette Sale, sur l'Oppius. Ce dessin, largement diffusé au XVIII ${ }^{\mathrm{e}}$ siècle, présente de grandes similitudes avec la peinture de Caylus. On pourrait imaginer que le Guerrier grec soit un fragment de cette frise mais il n'en est rien. Des analyses effectuées au laboratoire de la Bibliothèque nationale de France par Nathalie Buisson, ingénieur de recherche, ont montré que l'œuvre était un faux: la présence de grande quantité de blanc de plomb, un pigment peu utilisé en peinture murale romaine, a permis de confirmer nos doutes sur l'authenticité de cette œuvre ${ }^{11}$.

9 A. de Caylus, Recueil d'Antiquités, t. I, 1752, pl. LV, 1. Cette œuvre est aujourd'hui conservée au cabinet des Médailles.

10 Ibid.

11 Voir N. Buisson, D. Burlot, "La supercherie du faussaire Giuseppe Guerra", Techné, Brigitte Bourgeois éd., 28 (2008), p. 29-35. 
Elle a été réalisée par le faussaire Giuseppe Guerra, qui a fabriqué un grand nombre de contrefaçons de peinture antique dans les années 1750-1760 ${ }^{12}$.

La plus grande erreur de Caylus n'est sans doute pas de s'être laissé tromper, mais d'avoir publié l'œuvre sans contrôler son origine. Pourtant Taitbout, le consul de France à Naples, l'avait mis en garde sur les fausses peintures antiques d'Herculanum que l'on pouvait y acheter: «Bien que je m'assure que le morceau que ce religieux vous a procuré, soit bien tel qu'il vous l'a dit, et qu'on ne saurait vous en imposer; l'avis, au moins, pourra vous mettre encore plus sur vos gardes ${ }^{13}$. " Pourquoi Caylus a-t-il publié le guerrier grec malgré tout? Peut-être la volonté d'être le premier à publier une peinture d'Herculanum lui a-t-elle fait prendre un risque ${ }^{14}$. N'ayant jamais été à Herculanum, Caylus n'avait pas d'élément de comparaison et pouvait être facilement trompé. Et l'aubaine était sans doute trop importante pour la laisser passer!

Ses confrères s'aperçurent de son erreur et certains s'en moquèrent. Winckelmann relate l'histoire une dizaine d'années plus tard dans un ouvrage sur les découvertes d'Herculanum ${ }^{15}$. Caylus décide de traduire cet ouvrage de l'allemand au français. Caylus cherche à faire connaitre le livre de Winckelmann à une audience plus large que le seul public germanophone, et ce dessein semble louable. Or, il espère ainsi que les autorités napolitaines s'aperçoivent ainsi que Winckelmann brave leur interdiction en publiant des informations sur les découvertes, ce qui pourrait les offenser. Caylus ne rend donc pas service à Winckelmann en traduisant son livre, il lui rend l'accès à Herculanum plus difficile ${ }^{16}$. Il profite de la traduction pour corriger le texte, notam-

12 Giuseppe Guerra Veneziano (1709-1761) était peintre et graveur, mais il s'est rendu célèbre pour son activité de faussaire de peintures antiques. Entre 1750 et 1760 il réalisa près d'une centaine de contrefaçons imitant les peintures retrouvées à Herculanum et Pompéi. Il fut découvert en 1758 suite à une enquête de la cour napolitaine mais il ne fut pas véritablement inquiété car il était protégé par le Vatican. Il poursuivit son activité jusqu’à sa mort. Voir J.-J. BarthÉlemy, Voyage en Italie, Paris, 1802, p. 101-103, et ACCADEMIA Ercolanese, Le Antichità di Ercolano esposte, Naples, vol. II, 1760, préface (n. p.). Son autoportrait dessiné est conservé à Windsor, A. BLUNT, Venetian drawings of the XVII-XVIII centuries in the collection of Her Majesty the Queen at Windsor Castle, Londres, 1957, p. 27. Un tableau de lui réalisé en collaboration avec Canaletto représentant l'Etna en éruption a récemment été vendu aux enchères chez Christie's, Important Old Master Paintings, New York, 26 janvier 2001, lot $\mathrm{n}^{\circ} 165$.

13 Lettre de Taitbout au comte de Caylus datée du 8 novembre 1751, SERIEYs Antoine, Lettres inédites d'Henri IV et de plusieurs personnages célèbres, Paris, 1802, p. 207.

14 Il est possible également que Caylus ait reçu la mise en garde trop tard, alors que son ouvrage était déjà sous presse.

15 Der Herr Graf Caylus ließ eins dergleichen als ein altes Gemälde in seinen Sammlungen von Altherthümern stechen, weil man es ihm als ein Stück aus dem Herculano verkauft hatte, J.J. WincKelmann, Sendschreiben von den Herculanischen Entdeckungen, Dresde, 1762, p. 31.

16 E. Décultot, "Winckelmann et Caylus", in N. Cronk, K. Peeters (éd.), Le comte de Caylus, les arts et les lettres (colloque international, Université d'Anvers et Voltaire Foundation, Oxford, 2000), Amsterdam, New York, 2004, p. 62. 
ment le passage concernant le guerrier grec, auquel il ajoute cette note: "M. le comte de Caylus n'a rien fait graver de semblable, $\&$ bien loin de s'être laissé tromper, il a peut-être été le premier à parler de l'imposture de Guerra. Voyez ce qu'il a écrit sur le sujet dans le tome IV de son Recueil d'antiquités, p. $220^{17}$. " Caylus nie les faits et dans aucun de ses écrits il n'a admis avoir publié une ouvre de Guerra ${ }^{18}$. Il semble avoir honte de son erreur et il préfere l'occulter.

\section{La séduction de l'antiquaire}

Winckelmann, lorsqu'il relate l'affaire des peintures de Guerra, insiste sur le fait qu'il n'a jamais été dupé par le faussaire, cherchant ainsi à affirmer sa supériorité par rapport à Caylus. Or, cette même année, Giovanni Casanova, qui travaillait pour lui comme graveur, lui parla de peintures antiques très belles, conservées chez un collectionneur français, le chevalier Diel de Marsilly, résidant à Rome ${ }^{19}$. Casanova obtint pour Winckelmann une permission exceptionnelle d'aller voir l'une de ces œuvres, représentant Jupiter et Ganymède. Winckelmann fut conquis. Il fut émerveillé par le Ganymède, que selon lui « seul le pinceau de Mengs, le Raphaël moderne ${ }^{20}$ ", pourrait décrire. Le caractère homoérotique de la composition ne l'a sans doute pas laissé indifférent. Il publie dans la première édition de son Histoire de l'art un commentaire sur cette ouvre ${ }^{21}$.

Winckelmann publie également dans cet ouvrage deux autres peintures de la collection Diel de Marsilly, qu'il n'a pas vues, mais dont il connaît des gravures réalisées par Casanova, et mentionne quatre autres fragments, qui seraient passés en Angleterre à la mort de Diel. Les deux peintures dont il publie la gravure représentent l'Éducation d'Érichtonios (fig. 1) et des Ménades dansant ${ }^{22}$. Winckelmann est intéressé par

17 J. J. Winckelmann, Lettre de M. l'abbé Winckelmann à M. le comte de Brühl sur les découvertes d'Herculanum, Paris, 1764, p. 32, n. g.

18 La correspondance entre le père Paciaudi et le comte de Caylus fait de nombreuses références à l'histoire de Guerra. Paciaudi engage Caylus à publier l'une des peintures du faussaire afin de révéler la supercherie, mais Caylus refuse. "Je désirerais que vous missiez au jour une de ces peintures, celle qui est la plus parfaite et qui porte tous les caractères de la composition de Guerra. Dans trente ans d'ici, on ne connaîtra plus cette charlatanerie; on ignorera combien de personnes furent les dupes de leur bonne foi, et, qui pis est, on considèrera ces peintures comme antiques ", lettre de Paciaudi au comte de Caylus, Rome, le 2 septembre, 1760, Serieys Antoine, Lettres de Paciaudi au Comte de Caylus, Paris, H. Tardieu, 1802, p. 171.

19 Giovanni Casanova (1730-1795) est le frère cadet de Gian Giacomo Casanova. Formé comme peintre et graveur, il exerça à Rome à partir de 1752, et partit s'installer à Dresde en 1764 .

20 J. J. Winckelmann, Geschichte der Kunst des Alterthums, Walther, Dresde, 1764, p. 283.

21 Ibid., p. 283.

22 Ibid., p. 262 et 263. 
les costumes représentés dans ces peintures, et il pense qu'il pourrait s'agir d'œuvres très anciennes. Il est intéressé aussi par la représentation d'un mythe peu connu, qu'il se plaît à identifier. La provenance des peintures lui est inconnue mais il sait qu'elles ont été achetées à grand prix par le collectionneur. Le commentaire de Winckelmann sur ces peintures tient une place non négligeable dans son chapitre consacré à la peinture des Anciens. Or, peu après la parution de Geschichte der Kunst des Alterthums, Winckelmann s'aperçoit qu'il a été trompé par son graveur, Casanova, car celui-ci a tout inventé: les peintures n'ont jamais existé et les gravures que Casanova lui a présentées sortaient de son imagination. Winckelmann se mit fortement en colère lorsqu'il l'apprit et il fit détruire les cuivres afin que les œuvres ne soient pas publiées à nouveau ${ }^{23}$. Il les fit retirer de l'édition française de son ouvrage, alors en cours d'impression. Il se brouilla avec Casanova et chercha, sans succès, à l'envoyer aux galères pour une histoire de falsification de lettre de change.

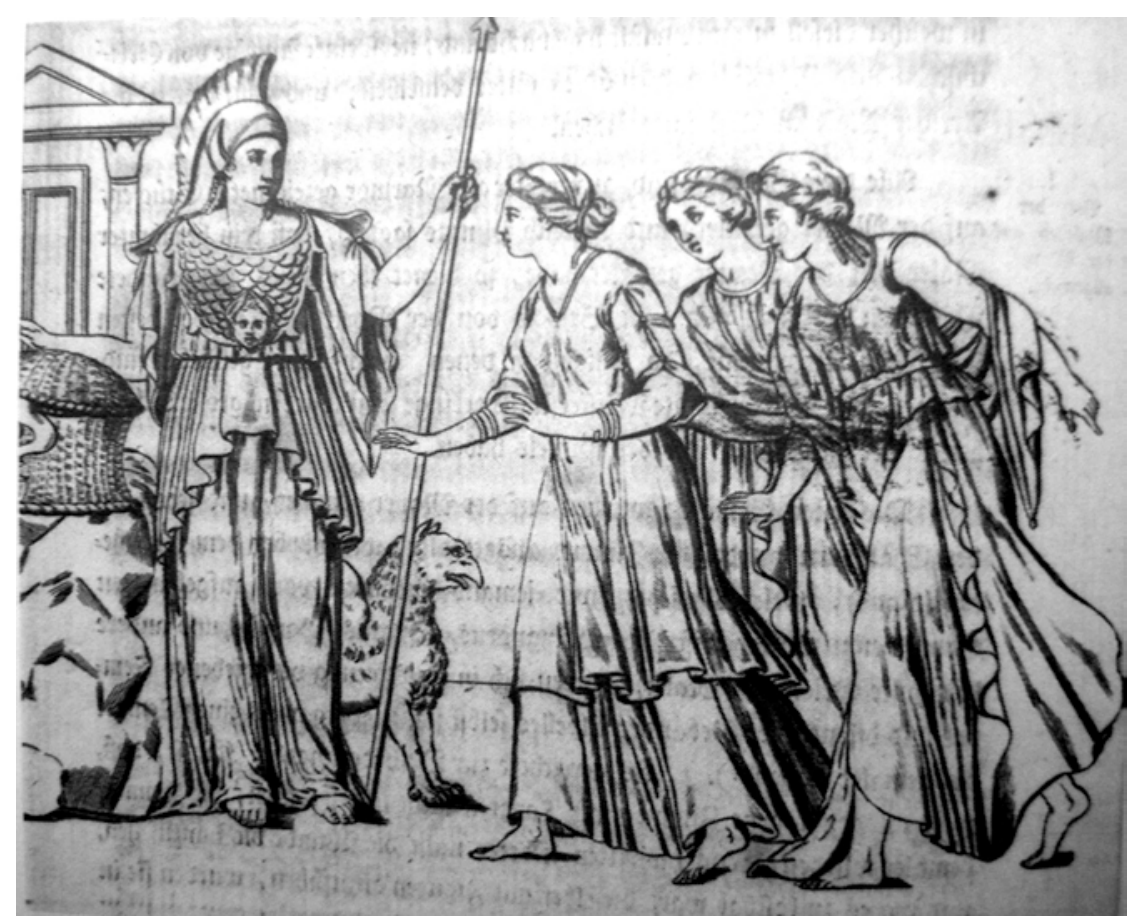

Fig. 1

23 Karl JusTI, Winckelmann sein Leben, seine Werke und seine Zeitgenossen, Leipzig, II, 1872, p. 304 ; l'édition française de Geschichte était en préparation lorsque Winckelmann s'aperçut de la supercherie. 
Winckelmann se brouilla également avec Mengs, probablement parce qu'il le soupçonnait d'avoir pris part à la supercherie. Il semblerait qu'il n'ait jamais su quel rôle avait joué le peintre, mais il savait qu'il n'était pas étranger à la farce. En réalité, plusieurs auteurs attribuent la peinture de Jupiter et Ganymède à Mengs, qui se serait inspiré des fresques de Raphaël à la Farnésine ${ }^{24}$. Azara, son biographe, nous apprend qu'il a fait de fausses retouches sur la peinture pour laisser croire qu'elle avait été restaurée après dépose ${ }^{25}$. Winckelmann n'a jamais su, ou n'a jamais voulu admettre que cette œuvre était un faux. La désillusion était sans doute trop grande.

Pourquoi Mengs et Casanova ont-ils cherché à duper leur ami et collègue? Mengs pensait que les peintures antiques avaient été réalisées à fresque et il s'opposait ainsi à Winckelmann qui croyait les peintures antiques exécutées à la détrempe ${ }^{26}$. Il est possible que Mengs ait décidé de peindre à fresque le Jupiter et Ganymède afin de le présenter à Winckelmann comme un original antique et ensuite lui faire accepter son hypothèse sur la technique de peinture des anciens ${ }^{27}$. Il a monté cette supercherie avec la complicité de Casanova, et il ne pensait sans doute pas qu'elle allait prendre autant d'ampleur. Devant la réaction enthousiaste de Winckelmann, les deux artistes ont eu du mal à lui avouer la vérité. On peut soupçonner Casanova de malice car il a fait durer la tromperie en produisant d'autres faux. Cela lui plaisait de duper Winckelmann, qui s'estimait supérieur aux autres antiquaires, et loin de l'erreur. Winckelmann est tombé dans le piège que Casanova et Mengs ont fabriqué pour lui. Dans le Jupiter et Ganymède, tout est conforme à un certain " fantasme " d'antiquaire, peut-on le blâmer d'y avoir succombé?

La déclaration de Winckelmann, disant qu'il n'avait jamais été dupé par Guerra doit être nuancée. Winckelmann publie en 1767 dans ses Monumenti Antichi Inediti, une peinture représentant une scène de sacrifice (fig. 2), conservée dans la collection du cardinal Albani pour lequel il travaille comme bibliothécaire ${ }^{28}$. Il voit dans cette œuvre la représentation de Livie et Octavie sacrifiant un Mars, et rapproche la scène d'un passage d'Horace. Winckelmann est surpris par le fait que Mars soit armé d'une massue, et qui plus est, d'une massue à pointes, « de celles dont se servaient les Assyriens

24 Une analyse de cette peinture se trouve dans S. RöTtGen (éd.), Mengs, la scoperta del neoclassico, (cat. exp. Padoue, Palazzo Zabarella, 2001, Dresde, Staatliche Kunstsammlungen, 2001), Venise, 2001, p. 242.

25 G.N. Azara, C. Fea, Opere di Antonio Raffaello Mengs, Rome, 1787, p. XXXII.

26 Nous savons aujourd'hui que la technique des peintres romains est un type de peinture à fresque. P. Philippot, L. Mora, P. Mora, La conservation des peintures murales, Bologne, 1977, p. 279-299.

27 Cette hypothèse pour expliquer la mise au point de la supercherie est donnée par Röttgen, op. cit. n. 24.

28 J.J. Winckelmann, Monumenti Antichi Inediti, Rome, 1821, p. 232, nº 177. 
et les Éthiopiens " (quella specie che era l'arme degli Assirj e degli Etiopi ${ }^{29}$ ), mais cela ne l'alerte pas et il ne semble pas douter de l'authenticité de l'œuvre.

Cette œuvre est proche stylistiquement du Guerrier grec, c'est donc probablement une peinture de Guerra. Il est possible que Winckelmann n'ait pas été dupe, mais qu'il ait publié cette œuvre pour faire plaisir au cardinal Albani. Goethe écrira en substance quelques années plus tard, à propos de la relation de ces deux hommes: « $S^{\prime}$ il (Winckelmann) vivait encore [...], il serait le premier à nous donner un remaniement de son travail. [...] Et puis, le cardinal Albani serait mort, par respect duquel il a écrit et peut-être dissimulé tant de choses ${ }^{30}$.»

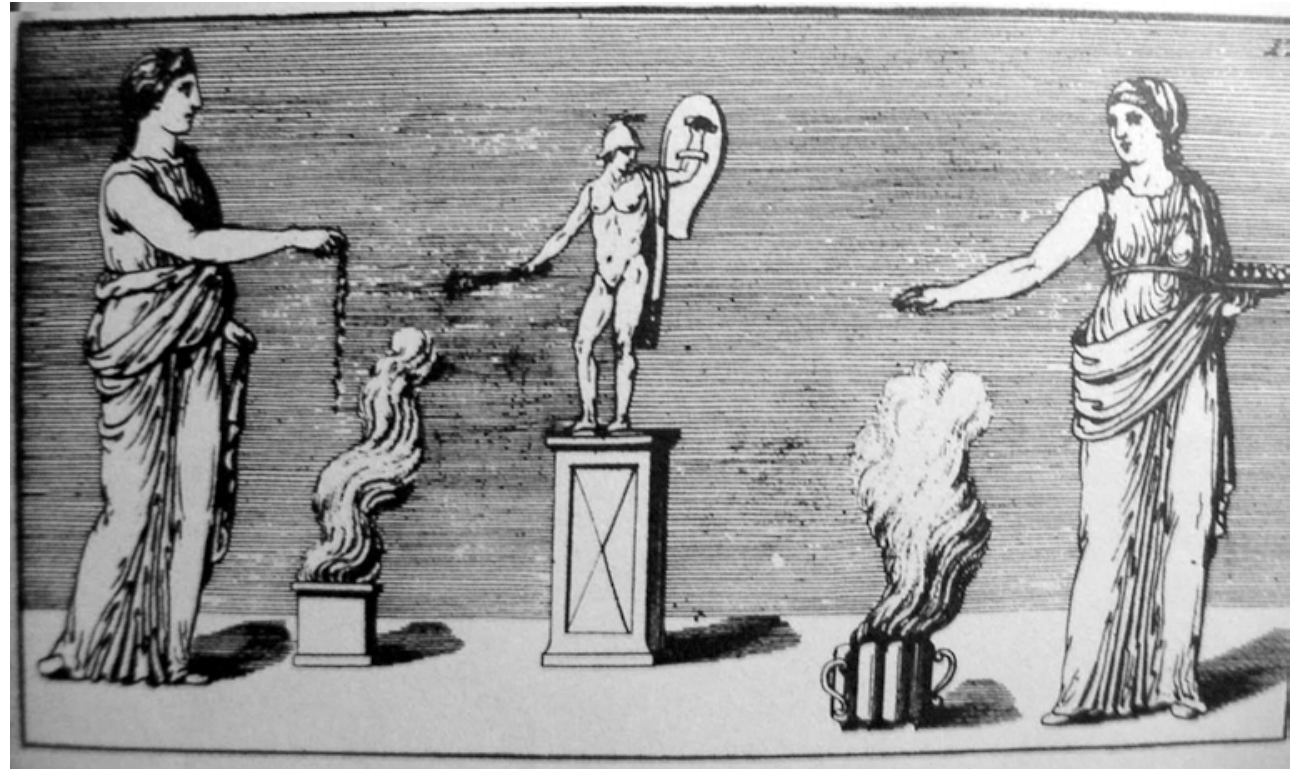

Fig. 2

\section{Inventer le passé}

En 1744 une peinture antique représentant la muse Polymnie fut présentée à l'Académie étrusque de Cortone ${ }^{31}$. Elle avait été trouvée par un paysan alors qu'il labourait son champ. Il l'avait prise pour une image de la Madone et l'avait placée au-dessus de

29 Ibid.

30 J. W. von Goethe, Voyage en Italie, Paris, 2003, p. 185.

31 Sur cette peinture, voir l'article de M. De Vos, "La Musa Polimnia di Cortona: una pittura pseudo-antica commissionata da Marcello Venuti", in P. Barocchi, D. Gallo (éd.), L'Accademia etrusca, (cat. exp. Cortone, Palazzo Casali, 1985), Cortone, 1985, p. 69-72. 
sa cheminée. Le propriétaire du champ, en visite chez son fermier, découvrit l'œuvre et reconnut une peinture antique. Il l'acheta au paysan et c'est ainsi qu'elle put être présentée à l'Académie étrusque de Cortone. Cette ouvre, à la différence des peintures de Pompéi, n'est pas une peinture murale. Il s'agit d'un pinax, une peinture de chevalet, et de plus, celle-ci est peinte sur un fragment d'ardoise. Les membres de l'Académie étrusque la jugèrent grecque... Il semblerait pourtant que cette œuvre ait une autre origine. Son apparition coïncide avec le retour de Marcello Venuti, éminent antiquaire, dans sa ville natale de Cortone. Venuti avait travaillé pour le roi de Naples et avait participé activement à l'organisation des fouilles d'Herculanum. Cependant, des inimitiés et la divulgation (à ses amis florentins) d'informations sur les découvertes le mirent dans une position inconfortable, qui le força à quitter sa fonction et rentrer à Cortone ${ }^{32}$.

Venuti cherchait à faire valoir le patrimoine de sa ville, est il est peut-être allé jusqu'à fabriquer les objets qui y manquaient. C'est en effet probablement à son initiative que la Muse de Cortone a été réalisée. La découverte d'une peinture de chevalet, grecque, exécutée sur ardoise, un matériau rare, de style classique, plus proche de Raphaël que d'Apelle, rendait le passé de la ville de Cortone plus noble et plus prestigieux que celui d'Herculanum, où l'on ne trouvait que des peintures murales, romaines et de "style médiocre ${ }^{33}$ ». En inventant la Muse de Cortone, Venuti a fabriqué un passé à sa ville et réalisé son désir d'antiquaire. La contrefaçon est une commande faite à un artiste, qu'il a pu rencontrer dans le cercle des restaurateurs ou des copistes travaillant à Herculanum. Il ne lui restait plus qu'à publier l'œuvre, ce qu'il fit, dans son ouvrage sur les découvertes campaniennes ${ }^{34}$. La Muse de Cortone figure en bonne place à côté des peintures des cités vésuviennes et Venuti souligne sa rareté et sa valeur historique. C'est probablement parce qu'elle a été réalisée avec la complicité d'un antiquaire, que la Muse tarda à être dévoilée. Au cours des XVIII ${ }^{\mathrm{e}}$, XIX et XXe siècles elle fut fréquemment admirée, citée en exemple et fut même... imitée.

Au début du XIX $X^{\mathrm{e}}$ siècle à Rome apparaît une autre peinture à l'encaustique sur ardoise, représentant cette fois-ci la reine Cléopâtre en train de se donner la mort par le serpent Naja. Elle aurait été découverte à la villa d'Hadrien à Tivoli, près du Canope

32 D. Gallo, "Marcello Venuti tra Napoli e Cortona", in P. Barocchi, D. Gallo (éd.), L'Accademia etrusca, (cat. exp. Cortone, Palazzo Casali, 1985), Cortone, 1985, p. 56.

33 Cette opinion était partagée par beaucoup. Les peintures d'Herculanum n'étaient pas appréciées pour leur beauté mais pour leur Antiquité. Voir C. Michel, "Les peintures d'Herculanum et la querelle des Anciens et des Modernes", Bulletin de la Société de l'Histoire de l'Art Français, 1984, p. 105-117.

34 «[... Non vi restando ne meno un vestigio nel mondo delle antiche dipinte tavole? se pure non volessimo eccettuarne una piera lavagna dipinta, che essendo stata ritrovata in un sotterraneo nel Territorio di Cortona alcuni anni sono ", M. Venuti, Descrizione delle prime scoperte dell'antica città d'Ercolano ritrovata vicino a Portici, Venise, 1748, p. 107-108. 
et elle serait la peinture qu'Auguste aurait mise sur son char lors de son triomphe de la bataille d'Actium ${ }^{35}$. Elle eut différents propriétaires dont le baron de Benneval qui à la fin du XIX ${ }^{\mathrm{e}}$ siècle tenta de la vendre à un grand musée européen. Malgré plusieurs articles élogieux sur l'œuvre dans la presse, ni le Louvre, ni le British Museum, ni l'Ermitage ne se portèrent acquéreurs. En réalité ces articles, écrits par des amis de Benneval, cherchaient surtout à accroître la valeur de cette "Cléopâtre " et à prouver qu'elle était authentique. John Sartain, peintre américain ayant séjourné plusieurs fois chez Benneval, écrit ainsi, dans une monographie sur l'œuvre: "Bien sûr il y a cette querelle habituelle, à savoir si cette peinture est antique ou non, car il y a toujours des gens qui s'amusent à jeter le doute sur presque tous les objets du passé, se souciant peu de la véracité des preuves en faveur de leur authenticité. Cependant même eux s'accordent pour croire que la Cléopâtre est une ouvre de la Renaissance. Toutefois, l'opinion générale des archéologues est en faveur de son antiquité. " (Of course there has been the usual discussion as to whether this painting is really antique, for there are always people of that peculiar propensity whose chief pleasure and amusement consist in throwing doubt on nearly everything of the past, no matter how well established by proof. But even these place it in the epoch of the great artists of the Renaissance. The general decision of the archaeologists, however, is in favor of its antiquity ${ }^{36}$.) Ainsi, selon lui, la Cléopâtre, malgré les doutes qui pèsent sur son authenticité, est digne d'intérêt, car si elle n'est pas antique, elle a été faite à la Renaissance. Sartain est dans l'erreur, puisque cette œuvre n'appartient à aucune des deux époques: elle a très probablement été réalisée à la fin du XVIII ${ }^{\mathrm{e}}$ siècle, alors que la peinture à l'encaustique connaissait un certain renouveau; on pensait alors que la plupart des peintures antiques et notamment les peintures de Pompéi, avaient été réalisées à la cire ${ }^{37}$. Plusieurs recettes avaient été mise au point par des théoriciens, et testées par des artistes ${ }^{38}$.

Cette mode de l'encaustique eut une conséquence fâcheuse: considérées comme peintes à la cire, les peintures romaines antiques furent vernies avec des produits à base de cire, ce qui fut fort préjudiciable pour leur conservation. De nombreuses peintures de Pompéi ont souffert de ces traitements.

Les faussaires reproduisirent cette erreur dans leurs œuvres: les contrefaçons de peinture antique qui apparaissent au début du XIX ${ }^{\mathrm{e}}$ siècle sont peintes à la cire, ce qui

35 Un ouvrage sur cette œuvre a été rédigé à la fin du XIX eiècle: R. SCHOENER, Peinture grecque ancienne à l'encaustique sur ardoise représentant la Reine Cléopatre se donnant la mort au moyen du serpent africain le "Naja ", Paris, 1889.

36 J. SARTAIN, On the antique painting in encaustic of Cleopatra: discovered in 1818, Philadelphie, 1885, p. 8.

37 Voir E. LeVI, "L'articolo sull'incausto di Ugo Foscolo", La Bibliofilia, vol. XV, 1914, p. 68-90.

38 Pour le renouveau de la technique de peinture à la cire, voir D. RiCE, The Fire of the Ancients: the Encaustic Painting Revival, 1755 to 1812, Ann Arbor, 1979. 
nous permet de les distinguer des peintures originales, peintes à fresque, et nous indique un repère chronologique.

La réalisation d'un faux ne se limite pas à sa fabrication, il s'agit d'inventer l'histoire d'un objet, répondant aux envies et aux aspirations d'un antiquaire. Le faux est destiné à séduire son acheteur. S’il l'induit en erreur, alors la supercherie est réussie. Le faussaire cherche souvent à prouver qu'il en sait plus que l'homme de lettres, tout en lui faisant plaisir. Lorsque le faux est dévoilé, l'antiquaire conçoit souvent une certaine honte à avoir été trompé et certaines contrefaçons, une fois découvertes, sont détruites. C'est dommage car les contrefaçons nous renseignent sur les goûts et les modes d'une époque, sur l'histoire de l'archéologie, et l'histoire des théories. Le faux est essentiel à la compréhension du contexte historique, il est temps de lui donner la place qui lui revient dans l'historiographie.

Delphine BurLot

Docteur en archéologie (université Paris IV)

20, rue Pépin

93100 Montreuil

delphine.burlot@free.fr 